\title{
Numerical Solution for an Epicycloid Crack
}

\author{
Nik Mohd Asri Nik Long, ${ }^{1,2}$ Koo Lee Feng, ${ }^{2,3}$ Wong Tze Jin,,3 and Z. K. Eshkuvatov,2 \\ ${ }^{1}$ Department of Mathematics, Universiti Putra Malaysia, 43400 Serdang, Selangor, Malaysia \\ ${ }^{2}$ Institute for Mathematical Research, Universiti Putra Malaysia, 43400 Serdang, Selangor, Malaysia \\ ${ }^{3}$ Department of Basic Science and Engineering, Faculty of Agriculture and Food Sciences, Universiti Putra Malaysia, \\ Sarawak Campus, 97008 Bintulu, Sarawak, Malaysia
}

Correspondence should be addressed to Koo Lee Feng; kooleefeng@yahoo.com

Received 22 March 2014; Accepted 6 June 2014; Published 14 July 2014

Academic Editor: Ray K.L. Su

Copyright (c) 2014 Nik Mohd Asri Nik Long et al. This is an open access article distributed under the Creative Commons Attribution License, which permits unrestricted use, distribution, and reproduction in any medium, provided the original work is properly cited.

\begin{abstract}
A flat crack, $\Omega$, is lying in a three-dimensional homogenous isotropic elastic solid subjected to shear loading. A mathematical formulation is developed based on the mixed boundary values for $\Omega$ such that the problem of finding the resulting force can be written in the form of hypersingular integral equation. Employing conformal mapping, the integral equation is transformed to a similar equation over a circular region, $D$. By making a suitable representation of hypersingular integral equation, the problem is reduced to solve a system of linear equations. Numerical solution for the shear stress intensity factors, maximum stress intensity, and strain energy release rate is obtained. Our results give an excellent agreement to the existing asymptotic solutions.
\end{abstract}

\section{Introduction}

Crack problems play an important role in engineering application due to the fact that the presence of cracks may compromise the strength and toughness of structures. Hence, great efforts [1-10] have been made in solving the crack problems and it has been widely investigated since the pioneer work by Sneddon [11] for a penny-shaped crack. Integral transform method to the solution of a Fredholm integral equation of second kind and numerical approach was implemented by Kassir $[12,13]$ in solving the rectangular crack problem, while the classic collocation and Galerkin methods were applied by Ioakimidis [14] for solving the plane crack problem subjected to normal load, whereas a perturbation analysis and the complex potential method [15] were performed by Cotterell and Rice [16] to obtain the stress intensity factors for the curved and kinked crack subject to arbitrary tractions in an explicit and simple form. Recently, $\mathrm{Wu}$ [17] proposed the dual boundary element method to solve the antiplane crack problem, whilst Georgiadis and Gourgiotis [18] advocated distributed dislocation technique in solving crack problems within Cosserat elasticity with constrained rotations. Motivated by the work of Lazzarin and
Zappalorto [19], Lazzarin et al. [20] investigated the stress fields close to a rectangular hole in a plate of finite thickness.

Ioakimidis [21] introduced the concept of finite-part integrals and derived the hypersingular integral equation for a flat crack subjected to tensile pressure, where the unknown function is the crack displacement discontinuity while the right-hand terms are the applied tractions on the crack faces. This equation can be numerically solved effectively by using the Gaussian quadrature rules for finite-part integral. Hence, this concept had been advocated widely for the solution of crack problem and some of them can be found in [22-26].

In this paper, the epicycloid crack problem is formulated into solving the hypersingular integral equation numerically for finding the stress intensity factors, maximum stress intensity and energy release rate for the crack subject to shear loading. Our computational results agree with the existing asymptotic solution.

\section{Statement of Problem and Basic Equations}

Consider an arbitrary shaped crack, $\Omega$, embedded in threedimensional unbounded isotropic elastic body, $\Gamma$. Let the Cartesian coordinate $(x, y, z)$ with origin $O$ and $\Omega$ lie in the 


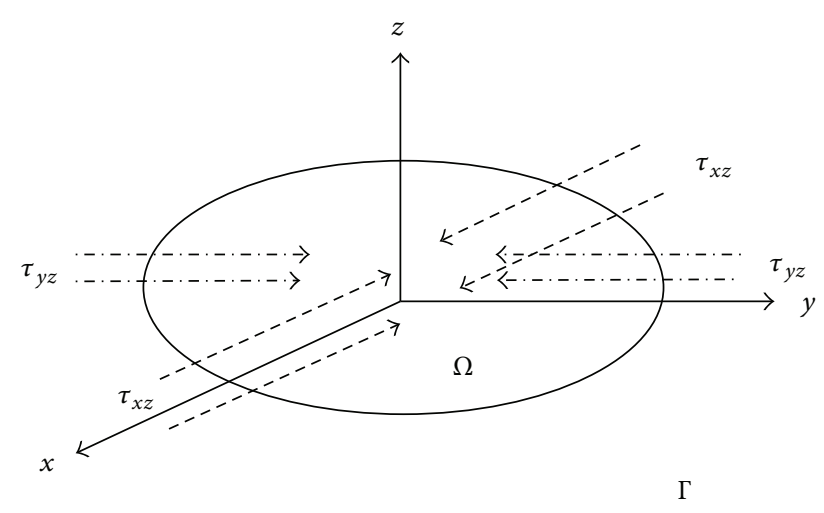

FIGURE 1: Stresses acting on a plane.

plane $z=0$. Assume that $O$ is a point in $\Omega$ and the body force is absent. Let the crack edges be deformed by the application of equal and opposite constant shear stresses in the $x$ and $y$ directions, $q_{x}(x, y)$ and $q_{y}(x, y)$, and it is assumed that the $z$ direction is traction-free; see Figure 1. Hence, in view of the shear load, the entire plane must be free from the normal stress; that is,

$$
\tau_{z z}=0 \quad \text { for } z=0
$$

Thus, the stress field can be found by considering the halfspace, $z \geq 0$, subject to the following mixed boundary condition on its surface $z=0$ :

$$
\begin{gathered}
\tau_{x z}=\frac{\mu}{1-\nu} q_{x}(x, y), \quad(x, y) \in \Omega, \\
\tau_{y z}=\frac{\mu}{1-\nu} q_{y}(x, y), \quad(x, y) \in \Omega, \\
u_{x}(x, y, z)=u_{y}(x, y, z)=0, \quad(x, y) \in \Gamma \backslash \Omega,
\end{gathered}
$$

where $\tau_{z z}, \tau_{x z}$, and $\tau_{y z}$ denote the stress tensor, $\mu$ is shear modulus, and $v$ is Poisson's ratio and the usual regularity requirements at the location away from the crack region,

$$
u_{i}(x, y, z)=O\left(\frac{1}{R}\right), \quad \tau_{i j}(x, y, z)=O\left(\frac{1}{R}\right),
$$

where $i, j=x, y, z, R \rightarrow \infty, R=\sqrt{\left(x-x_{0}\right)^{2}+\left(y-y_{0}\right)^{2}}, u_{i}$ are displacement vectors and $\tau_{i j}$ is given by

$$
\tau_{i j}=c_{i j k l} \frac{\partial u_{l}}{\partial x_{k}} ; \quad k, l=x, y, z
$$

where $c_{i j k l}$ are the material moduli defined by

$$
c_{i j k l}=\lambda \delta_{i j} \delta_{k l}+\mu\left(\delta_{i k} \delta_{j l}+\delta_{i l} \delta_{j k}\right),
$$

where $v=\lambda / 2(\lambda+\mu)$. The $\delta_{i j}$ is the Kronecker delta, defined as 1 if $i=j$ and 0 if $i \neq j$. And (5) must satisfy Hooke's law linear elasticity symmetry conditions:

$$
c_{i j k l}=c_{j i k l}=c_{k l i j}=c_{i j l k} .
$$

The displacement vector, $u_{i}$, is represented by Somigliana formula $[27,28]$ :

$$
u_{m}\left(x_{0}, y_{0}\right)=\int_{\Omega}\left[u_{i}(x, y)\right] \sum_{i j m}^{F}\left((x, y) ;\left(x_{0}, y_{0}\right)\right) n_{j} d \Omega,
$$

where the component of Green function is

$$
\begin{gathered}
\sum_{i j m}^{F}\left((x, y) ;\left(x_{0}, y_{0}\right)\right)=c_{i j k l} \frac{\partial}{\partial x_{k}} G_{l m}^{F}\left((x, y) ;\left(x_{0}, y_{0}\right)\right), \\
8 \pi \mu G_{i j}^{F}\left((x, y) ;\left(x_{0}, y_{0}\right)\right)=\frac{1}{8 \pi \mu}\left(\frac{2}{R} \delta_{i j}-\frac{1}{2(1-v)} \frac{\partial^{2} R}{\partial x_{i} \partial x_{j}}\right),
\end{gathered}
$$

and $\left[u_{i}(x, y)\right]$ is the displacement discontinuity in $u_{i}$ across the crack,

$$
\begin{aligned}
{\left[u_{i}(x, y)\right]=} & \lim _{\left(x_{0}, y_{0}\right) \rightarrow(x, y) \in \Omega^{+}} u_{i}\left(x_{0}, y_{0}\right) \\
& -\lim _{\left(x_{0}, y_{0}\right) \rightarrow(x, y) \in \Omega^{-}} u_{i}\left(x_{0}, y_{0}\right),
\end{aligned}
$$

where $n_{j}$ is the unit normal vector, which is assumed to point into $\Omega$. Equation (9) is known as Kelvin's point-load solution. Substitute (5) and (9) into (7) and perform the integration by parts with respect to $x$ and $y$, yielding a system of Cauchy principle-value integral equations [29]:

$$
\begin{aligned}
& q_{x}\left(x_{0}, y_{0}\right)=\frac{-1}{4 \pi} f_{\Omega}\left\{\alpha \frac{\partial}{\partial x}\left(\frac{1}{R}\right)+\beta \frac{\partial}{\partial y}\left(\frac{1}{R}\right)\right\} d \Omega, \\
& q_{y}\left(x_{0}, y_{0}\right)=\frac{-1}{4 \pi} f_{\Omega}\left\{\alpha \frac{\partial}{\partial y}\left(\frac{1}{R}\right)-\beta \frac{\partial}{\partial x}\left(\frac{1}{R}\right)\right\} d \Omega
\end{aligned}
$$

for $\left(x_{0}, y_{0}\right) \in \Omega$, where

$$
\alpha=\frac{\partial\left[u_{x}\right]}{\partial x}+\frac{\partial\left[u_{y}\right]}{\partial y}, \quad \beta=(1-v)\left(\frac{\partial\left[u_{x}\right]}{\partial y}-\frac{\partial\left[u_{y}\right]}{\partial x}\right) \text {. }
$$

The resulting boundary terms which involved $\left[u_{x}(x, y)\right]$ and $\left[u_{y}(x, y)\right]$ are evaluated at the crack edge, assuming that there are no tractions applied onto the boundary. Consequently, $\left[u_{x}(x, y)\right]$ and $\left[u_{y}(x, y)\right]$ are zero; that is, these equations are to be solved subject to

$$
\left[u_{x}(x, y)\right]=0, \quad\left[u_{y}(x, y)\right]=0 \quad \text { for }(x, y) \in \partial \Omega,
$$

where $\partial \Omega$ is the boundary of $\Omega$. Integrating (11) by parts and using condition (13) and making use of the relationship between Cauchy principle-value integral and hypersingular integral equations [30, 31],

$$
\frac{d}{d x} \int_{a}^{b} \frac{f(t)}{(x-t)} d t=-\int_{a}^{b} \frac{f(t)}{(x-t)^{2}} d t, \quad t \in(a, b),
$$

yield [29, 32]

$$
\begin{aligned}
q_{x} & \left(x_{0}, y_{0}\right) \\
& =\frac{1}{8 \pi} \gtrless_{\Omega} \frac{(2-\nu+3 \nu \cos 2 \Theta)\left[u_{x}\right]+3 \nu \sin 2 \Theta\left[u_{y}\right]}{8 \pi R^{3}} d \Omega,
\end{aligned}
$$




$$
\begin{aligned}
& q_{y}\left(x_{0}, y_{0}\right) \\
& \quad=\frac{1}{8 \pi} \oint_{\Omega} \frac{3 \nu \sin 2 \Theta\left[u_{x}\right]+(2-v-3 v \cos 2 \Theta)\left[u_{y}\right]}{R^{3}} d \Omega,
\end{aligned}
$$

and the angle $\Theta$ is defined by $x-x_{0}=R \cos \Theta$ and $y-y_{0}=$ $R \sin \Theta$. The cross on the integral of (15) and (16) means the hypersingular, and it must be interpreted as a Hadamard finite part integral $[31,33,34]$. Multiplying (16) with complex $j$ and adding to (15) lead to

$$
\begin{array}{r}
q\left(x_{0}, y_{0}\right)=\frac{1}{8 \pi} f_{\Omega} \frac{(2-v) w(x, y)+3 v e^{2 j \Theta} \overline{w(x, y)}}{R^{3}} d \Omega, \\
\left(x_{0}, y_{0}\right) \in \Omega,
\end{array}
$$

where $q\left(x_{0}, y_{0}\right)=q_{x}\left(x_{0}, y_{0}\right)+j q_{y}\left(x_{0}, y_{0}\right), w(x, y)=\left[u_{x}\right]+$ $j\left[u_{y}\right]$ is the unknown crack opening displacement, and the bar denotes the conjugation of $\overline{w(x, y)}=\left[u_{x}\right]-j\left[u_{y}\right]$ and $j^{2}=$ -1 . Equation (17) is to be solved subject to $w=0$ on $\partial \Omega$ and can be used for general crack problems under shear loading which is equivalent to those equations obtained in $[27,35]$. Suppose the constant shear stress is applied on opposite crack surfaces at $x$ direction, and then the general solution of (17) can be reduced into a single hypersingular integral equation:

$$
\begin{array}{r}
q\left(x_{0}, y_{0}\right)=\frac{1}{8 \pi} \oint_{\Omega} \frac{2-v+3 v e^{2 j \Theta}}{R^{3}} w(x, y) d \Omega \\
\left(x_{0}, y_{0}\right) \in \Omega .
\end{array}
$$

\section{Conformal Mapping and Epicycloid Cracks}

Suppose that $\Omega$ is a penny-shaped crack, with radius $a$ so that the crack occupies the region

$$
\Omega=\{(r, \theta): 0 \leq r<a,-\pi \leq \theta<\pi\},
$$

where $r$ and $\theta$ are polar coordinates, $x=r \cos \theta$, and $y=$ $r \sin \theta$.

Now, let $\Omega$ be a simply connected domain in the $z$-plane defined as

$$
\Omega=\{(r \cdot \theta): 0 \leq r<\rho(\theta),-\pi \leq \theta<\pi\}
$$

whose boundary has the polar equation $r=1+c \rho(\theta)$, where $\rho(\theta)$ is bounded and piecewise continuous and $c$ is a small positive parameter. Define $\zeta=s e^{i \phi}$ with $|\zeta|<1$ such that the circular unit disc, $D$, is defined as

$$
D \equiv\{(s, \phi): 0 \leq s<1,-\pi \leq \phi<\pi\} .
$$

Using the properties of Riemann Mapping theorem [36], a circular disc $D$ is mapped conformally onto $\Omega$ by

$$
z=a f(\zeta) \text { for }|\zeta|<1,
$$

where $\zeta=\xi+i \eta=s e^{i \phi}, \zeta_{0}=\xi_{0}+i \eta_{0}=s_{0} e^{i \phi_{0}}, x=a u(\xi, \eta)$, and $y=a v(\xi, \eta)$. Let

$$
\begin{gathered}
w(x(\zeta), y(\zeta))=a\left|f^{\prime}(\zeta)\right|^{-1 / 2} e^{j \delta} W(\xi, \eta), \\
q\left(x\left(\zeta_{0}\right), y\left(\zeta_{0}\right)\right)=a\left|f^{\prime}\left(\zeta_{0}\right)\right|^{-3 / 2} e^{j \delta_{0}} Q\left(\xi_{0}, \eta_{0}\right),
\end{gathered}
$$

and the analytic function $f$ in (22) is known to exist for any simply connected domain $\Omega$. Further, we assume that $\left|f^{\prime}(\zeta)\right|$ is nonzero and bounded for all $|\zeta|<1$. Define $S, \Phi, \delta$, and $\delta_{0}$ as

$$
\begin{gathered}
S e^{i \Phi}=\zeta-\zeta_{0}, \quad f^{\prime}(\zeta)=\left|f^{\prime}(\zeta)\right| e^{i \delta}, \\
f^{\prime}\left(\zeta_{0}\right)=\left|f^{\prime}\left(\zeta_{0}\right)\right| e^{i \delta_{0}} .
\end{gathered}
$$

Let $z-z_{0}=a\left(f(\zeta)-f\left(\zeta_{0}\right)\right)=R e^{i \Theta}$ such that, for small $S$, $R \simeq a S\left|f^{\prime}\left(\zeta_{0}\right)\right|$ and $\Theta \simeq \Phi+\delta_{0}$.

A similar integral equation with (18) can be obtained by substituting (23) and (24) into (18); that is,

$$
\begin{aligned}
Q\left(\xi_{0}, \eta_{0}\right)= & \frac{2-\nu+3 v e^{2 j \Theta}}{8 \pi} \oiint_{D} \frac{W(\xi, \eta)}{S^{3}} d \xi d \eta \\
& +\frac{2-\nu}{8 \pi} f_{D} W(\xi, \eta) K^{(1)}\left(\zeta, \zeta_{0}\right) d \xi d \eta \\
& +\frac{3 v}{8 \pi} \int_{D} W(\xi, \eta) K^{(2)}\left(\zeta, \zeta_{0}\right) d \xi d \eta ; \quad\left(\xi_{0}, \eta_{0}\right) \in D
\end{aligned}
$$

where $K^{(1)}\left(\zeta, \zeta_{0}\right)$ and $K^{(2)}\left(\zeta, \zeta_{0}\right)$ are Cauchy type singular and weak singular kernel, respectively [29]:

$$
\begin{aligned}
K^{(1)}\left(\zeta, \zeta_{0}\right) & =\frac{\left|f^{\prime}(\zeta)\right|^{3 / 2}\left|f^{\prime}\left(\zeta_{0}\right)\right|^{3 / 2}}{\left|f(\zeta)-f\left(\zeta_{0}\right)\right|^{3}} e^{j\left(\delta-\delta_{0}\right)}-\frac{1}{\left|\zeta-\zeta_{0}\right|^{3}}, \\
K^{(2)}\left(\zeta, \zeta_{0}\right) & =\frac{\left|f^{\prime}(\zeta)\right|^{3 / 2}\left|f^{\prime}\left(\zeta_{0}\right)\right|^{3 / 2}}{\left|f(\zeta)-f\left(\zeta_{0}\right)\right|^{3}} e^{j\left(2 \Theta-\delta-\delta_{0}\right)}-\frac{1}{\left|\zeta-\zeta_{0}\right|^{3}} e^{2 j \Phi} .
\end{aligned}
$$

This transformed hypersingular integral equation (25) over a circular disc $D$ is solved subject to $W=0$ on $s=1$.

\section{Numerical Treatment}

Define

$$
\begin{aligned}
& A_{k}^{n}(s, \phi)=s^{|n|} C_{2 k+1}^{|n|+(1 / 2)}\left(\sqrt{1-r^{2}}\right) e^{j n \phi}, \\
& L_{h}^{m}(s, \phi)=s^{|m|} C_{2 h+1}^{|m|+(1 / 2)}\left(\sqrt{1-r^{2}}\right) \cos m \phi
\end{aligned}
$$

such that the orthogonal polynomials $A_{k}^{n}(s, \phi)$ and $L_{h}^{m}(s, \phi)$ are satisfying the following relationship [37, Page 1054, 8.939.8]:

$$
\int_{\Omega} A_{k}^{n}(s, \phi) L_{h}^{m}(s, \phi) \frac{s d s d \phi}{\sqrt{1-s^{2}}}=B_{k}^{n} \delta_{k h} \delta_{m n},
$$




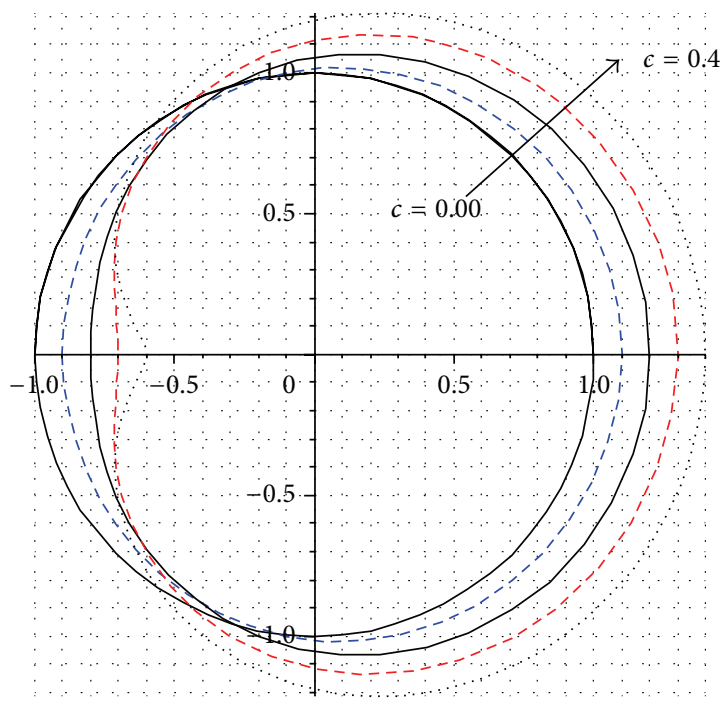

(a) $m=1$

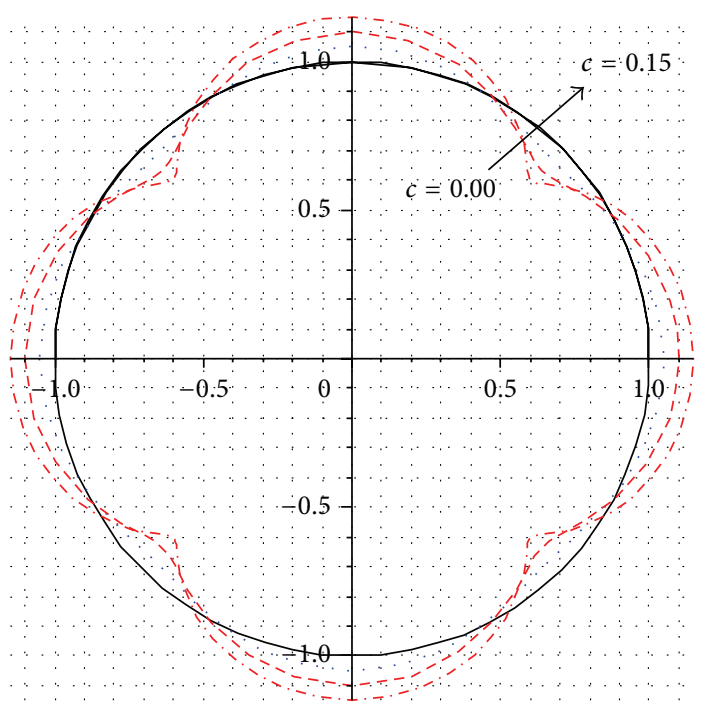

(b) $m=4$

FIGURE 2: The domain of $f(\zeta)$ with various $m$ and $c$.

TABLE 1: Numerical convergence for the sliding mode stress intensity factor, $K_{2}(\phi)$, for $m=1$ when $c=0.1$.

\begin{tabular}{cccccc}
\hline$N$ & $K_{2}(0.00)$ & $K_{2}(\pi / 4)$ & $K_{2}(\pi / 2)$ & $K_{2}(3 \pi / 4)$ & $K_{2}(\pi)$ \\
\hline 0 & $1.0423 E-03$ & $7.5379 E-04$ & $6.9319 E-20$ & $-8.6663 E-04$ & -1.6978 \\
1 & 1.4538 & 0.9883 & $6.7158 E-17$ & -0.9971 & -0.9411 \\
2 & 1.3545 & 0.8915 & $6.7071 E-17$ & -0.9716 & -0.9427 \\
3 & 1.3333 & 0.8914 & $6.4321 E-15$ & -0.9709 & -1.2468 \\
4 & 1.3191 & 0.8637 & -0.1194 & -0.9709 & -1.2468 \\
5 & 1.3191 & 0.8637 & -0.1194 & -0.9709 & -1.2468 \\
6 & 1.3191 & 0.8637 & -0.1194 & & \\
\hline
\end{tabular}

where the respective weight function is $w(s)=(1-s)^{-1 / 2}$ and

$$
\begin{array}{ll}
B_{k}^{n} & \begin{cases}\frac{\pi^{2} \Gamma(2 k+2)}{(2 k+(3 / 2))(2 k+1) ![\Gamma(1 / 2)]^{2}}, & n=0 \\
\frac{\pi^{2} \Gamma(2 k+2 n+2)}{2^{2 n+1}(2 k+n+(3 / 2))(2 k+1) ![\Gamma(n+(1 / 2))]^{2}}, & n \neq 0 .\end{cases}
\end{array}
$$

Write $W(\xi, \eta)$ as a finite sum

$$
W(\xi, \eta)=\sum_{n=-N_{1}}^{N_{1}} \sum_{k=0}^{N_{2}} s^{|n|} W_{k}^{n} C_{2 k+1}^{|n|+(1 / 2)}\left(\sqrt{1-s^{2}}\right) e^{j n \phi}
$$

Substituting (30) into (25) yields

$$
\sum_{n, k} \mathscr{F}_{k}^{n}\left(s_{0}, \phi_{0}\right) W_{k}^{n}=Q\left(\xi_{0}\left(s_{0}, \phi_{0}\right), \eta_{0}\left(s_{0}, \phi_{0}\right)\right)
$$

where

$$
\begin{aligned}
& \mathscr{F}_{k}^{n}\left(s_{0}, \phi_{0}\right)=-E_{k}^{n} \frac{\left(2-v+3 v e^{2 j \Theta}\right) A_{k}^{n}\left(s_{0}, \phi_{0}\right)}{2 \sqrt{1-s_{0}^{2}}} \\
&+\frac{2-v}{8 \pi} \int_{D} A_{k}^{n}(s, \phi) K^{(1)}\left(\zeta, \zeta_{0}\right) d \xi d \eta \\
&+\frac{3 v}{8 \pi} \int_{D} A_{k}^{n}(s, \phi) K^{(2)}\left(\zeta, \zeta_{0}\right) d \xi d \eta ; \\
& 0 \leq s \leq 1, \quad 0 \leq \phi<2 \pi .
\end{aligned}
$$

The following formula [38] is useful in deriving (31):

$$
\frac{1}{4 \pi} \oiint_{\Omega} \frac{A_{k}^{n}(s, \phi)}{R^{3}} d \Omega=-E_{k}^{n} \frac{A_{k}^{n}\left(s_{0}, \phi_{0}\right)}{\sqrt{1-s_{0}^{2}}}
$$

where

$$
E_{k}^{n}=\frac{\Gamma(|n|+k+(3 / 2)) \Gamma(k+(3 / 2))}{(|n|+k) ! k !} .
$$


TABLE 2: Numerical convergence for the sliding mode stress intensity factor, $K_{2}(\phi)$, for $m=1$ when $c=0.45$.

\begin{tabular}{lccccc}
\hline$N$ & $K_{2}(0.00)$ & $K_{2}(\pi / 4)$ & $K_{2}(\pi / 2)$ & $K_{2}(3 \pi / 4)$ & $K_{2}(\pi)$ \\
\hline 0 & $5.9156 E-04$ & $4.3514 E-04$ & $4.35045 E-20$ & $-6.7348 E-04$ & $-1.2782 E-03$ \\
1 & 0.0000 & 1.0959 & $3.1941 E-19$ & $-8.7363 E-03$ & $-3.3449 E-02$ \\
2 & 1.7569 & 1.0959 & $6.3681 E-17$ & -1.3444 & -0.3384 \\
3 & 1.7624 & 1.0930 & $6.3681 E-17$ & -1.3511 & -1.3057 \\
4 & 1.0945 & $6.3681 E-17$ & -1.3518 & -1.3020 \\
5 & 1.5604 & 0.9940 & $6.3681 E-17$ & -1.3518 & -1.3002 \\
6 & 1.4613 & 0.9812 & $6.3681 E-17$ & -1.3517 & -1.3993 \\
7 & 1.4609 & 0.8127 & -0.6744 & -1.7515 & -1.2989 \\
9 & 1.4011 & 0.8115 & -0.6744 & -1.9999 & -1.4986 \\
10 & 1.3610 & 0.8029 & -0.6317 & -1.1489 & -1.2984 \\
11 & 1.2030 & 0.7846 & -0.6000 & -1.1317 & -1.1983 \\
12 & 1.1948 & 0.7796 & -0.5913 & -1.1245 & -1.1203 \\
13 & 1.1849 & 0.6912 & -0.5410 & -1.0982 & -1.1782 \\
14 & 1.1782 & 0.5680 & -0.5302 & -1.0982 & -1.1782 \\
15 & 1.1782 & 0.5680 & -0.5302 & -1.0982 & -1.1782 \\
17 & 1.1782 & 0.5680 & -0.5302 & -0.5302 & \\
16 & 1.1782 & 0.5680 & & & \\
\hline
\end{tabular}

TABLE 3: Numerical convergence for the tearing mode stress intensity factor, $K_{3}(\phi)$, for $m=-2$ when $c=0.1$.

\begin{tabular}{lccccc}
\hline$N$ & $K_{3}(0.00)$ & $K_{3}(\pi / 4)$ & $K_{3}(\pi / 2)$ & $K_{3}(3 \pi / 4)$ & $K_{3}(\pi)$ \\
\hline 0 & 0.0000 & $-6.330 E-04$ & $-9.5075 E-04$ & $-7.2780 E-04$ & $-1.3145 E-19$ \\
1 & 0.0000 & -0.7174 & -0.9211 & -0.5854 & $-9.6785 E-17$ \\
2 & 0.0000 & -0.5440 & -0.9199 & -0.5854 & $-9.6951 E-17$ \\
3 & 0.0000 & -0.5200 & -0.8775 & -0.200 & $-1.04460 E-16$ \\
4 & 0.0000 & -0.5200 & -0.8775 & -0.5200 & $-1.04460 E-16$ \\
5 & 0.0000 & -0.5200 & -0.8775 & -0.5200 & $-1.04460 E-16$ \\
6 & 0.0000 & -0.5200 & -0.8775 & -0.5200 & $-1.04460 E-16$ \\
\hline
\end{tabular}

To determine the unknown coefficients, $W_{k}^{n}$, multiply (31) by $L_{h}^{m}\left(s_{0}, \phi_{0}\right)$ and integrate over $D$ and using (28), leads to

$$
\begin{aligned}
& \sum_{n, k} W_{k}^{n}\left(-\frac{2-\nu+3 v e^{2 j \Theta}}{2} \delta_{h k} \delta_{|m||n|}\right. \\
& +\frac{1}{8 \pi} \int_{D} L_{h}^{m}\left(\zeta_{0}\right) \int_{D} A_{k}^{n}(\zeta) \\
& \times\left[(2-\nu) K^{(1)}\left(\zeta, \zeta_{0}\right)\right. \\
& \left.\left.+3 \nu K^{(2)}\left(\zeta, \zeta_{0}\right)\right] d \zeta d \zeta_{0}\right) \\
& =\int_{D} Q\left(\zeta_{0}\right) L_{h}^{m}\left(\zeta_{0}\right) d \zeta_{0} ; \quad-N_{1} \leq m \leq N_{1}, 0 \leq h \leq N_{2}
\end{aligned}
$$

with the following notations:

$$
\begin{gathered}
\zeta_{0}=\zeta_{0}\left(s_{0}, \phi_{0}\right), \quad d \zeta_{0}=s_{0} d s_{0} d \phi_{0}, \\
Q\left(\zeta_{0}\right)=Q\left(\xi_{0}, \eta_{0}\right)=Q\left(s_{0} \cos \phi_{0}, s_{0} \sin \phi_{0}\right) .
\end{gathered}
$$

In evaluating the multiple integral in (35), we have used the Gaussian quadrature and trapezoidal formulas for the radial and angular directions with appropriate choice of collocation points $(s, \phi)$ and $\left(s_{0}, \phi_{0}\right)$. This effort leads to the $\left(2 N_{1}+\right.$ $1)\left(N_{2}+1\right) \times\left(2 N_{1}+1\right)\left(N_{2}+1\right)$ system of linear equations, $A w=b$ for the unknown coefficients of $W_{k}^{n}$, where $A=$ $\left(a_{i j}\right)$ is a square matrix and $w$ and $b$ are vectors, and, solved numerically using LAPACK routine F07ASF (ZGETRS) in Numerical Algorithms Group (NAG).

\section{Stress Intensity Factors, Maximum Stress Intensity, and Energy Release Rate}

The sliding mode, $K_{2}(\phi)$, and the tearing mode, $K_{3}(\phi)$, stress intensity factors are defined as $[39,40]$

$$
K_{j}(\phi)=\lim _{r \rightarrow a} \sqrt{\frac{2 \pi}{a-r}} V_{j} w(x, y) ; \quad j=2,3,
$$

where $V_{j}$ are constants. The maximum stress intensity, $M(\phi)$, is defined as

$$
M(\phi)=\sqrt{\left[K_{2}(\phi)\right]^{2}+\left[K_{3}(\phi)\right]^{2}}
$$




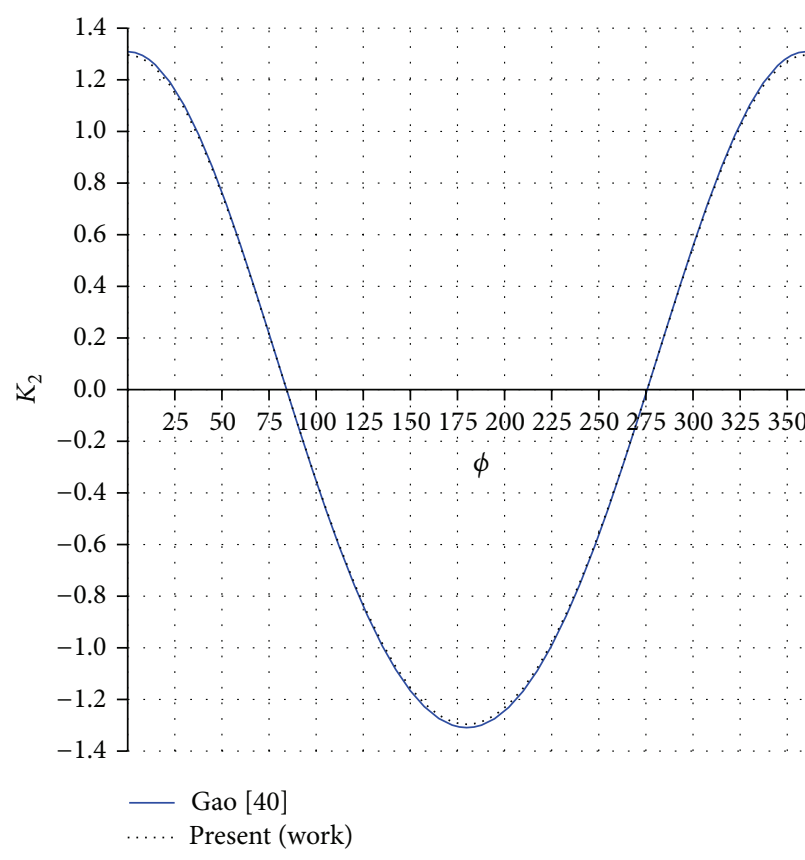

(a) $c=0.1$

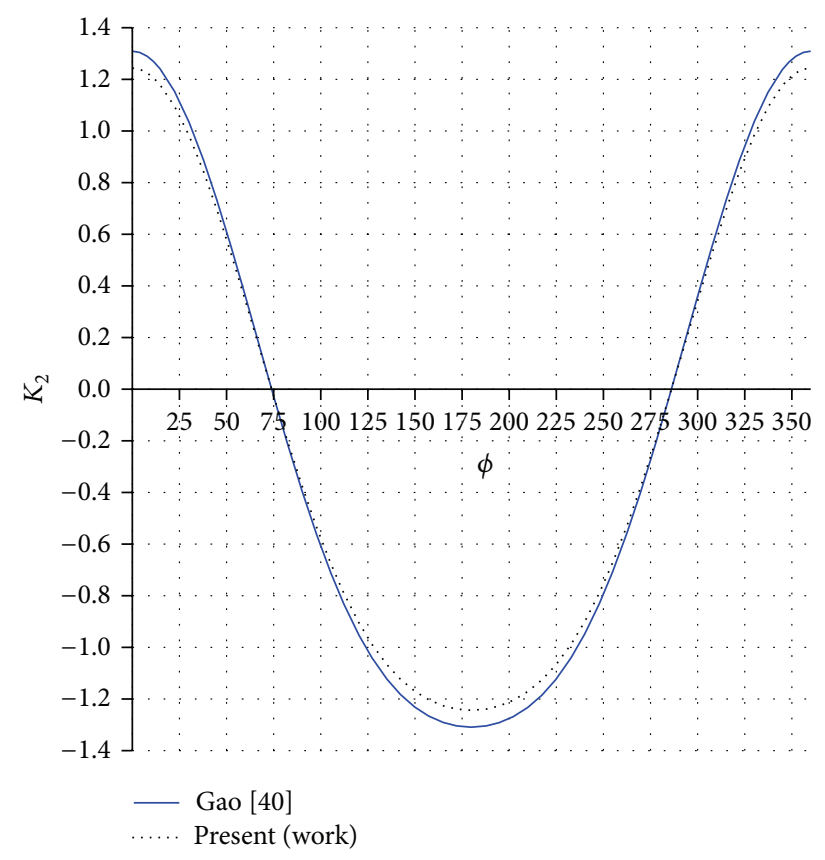

(b) $c=0.3$

Figure 3: The $K_{2}(\phi)$ for $f(\zeta)=\zeta+c \zeta^{2}$ at different $c$.

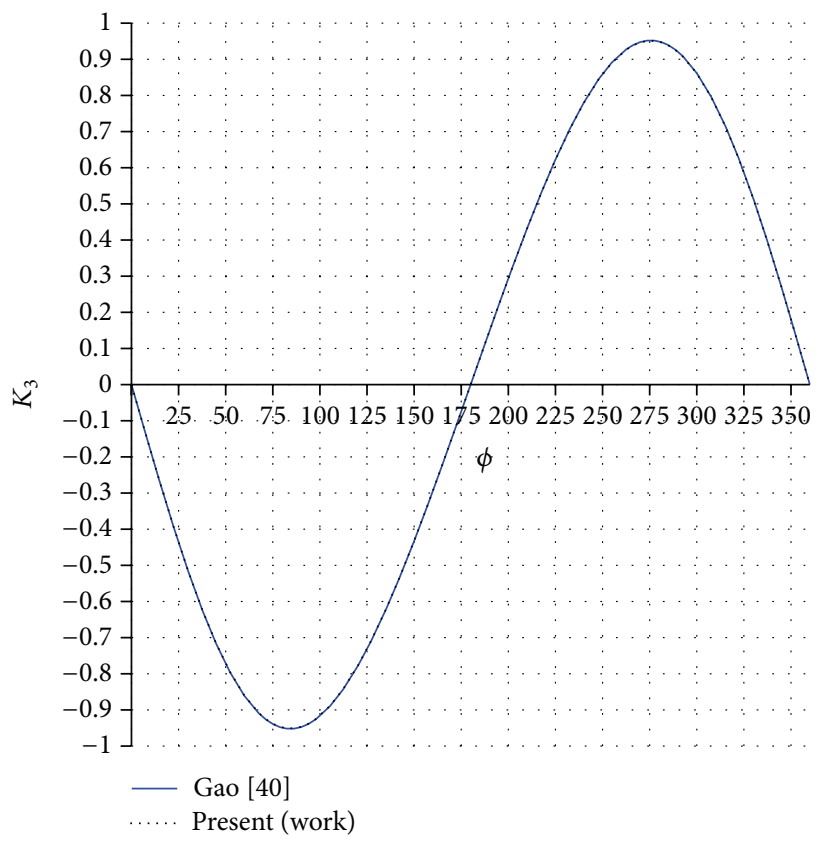

(a) $c=0.1$

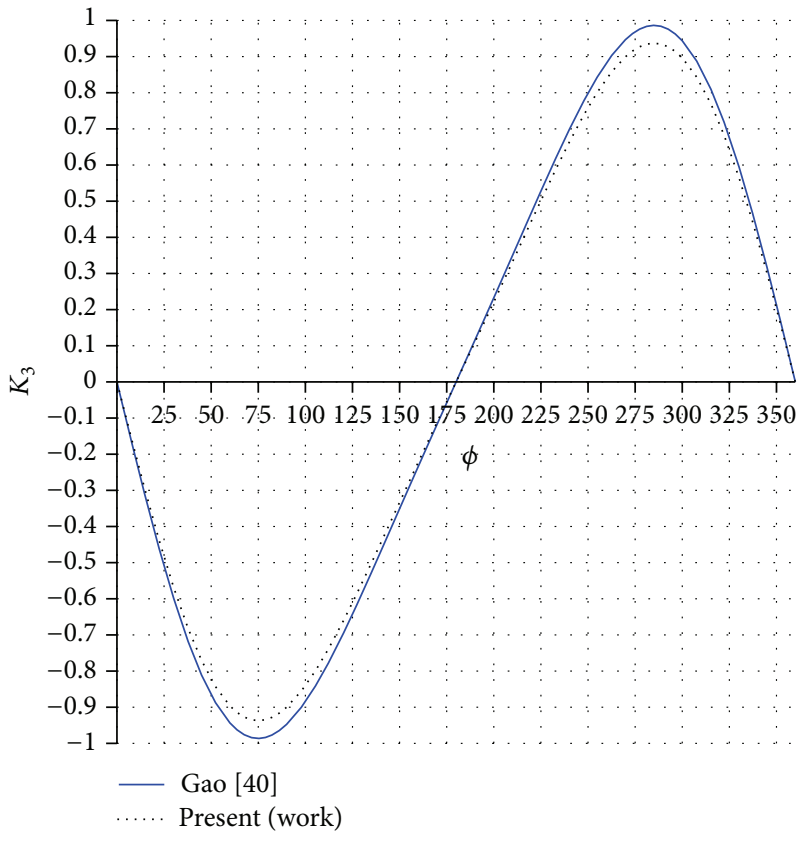

(b) $c=0.3$

Figure 4: The $K_{3}(\phi)$ for $f(\zeta)=\zeta+c \zeta^{2}$ at different $c$.

while the energy release rate, $G(\phi)$, by Irwin's relation subjected to shear loading is calculated from stress intensity factors and defined as

$$
G(\phi)=\frac{\left(1-v^{2}\right)}{E}\left[K_{2}(\phi)\right]^{2}+\frac{(1+\nu)}{E}\left[K_{3}(\phi)\right]^{2},
$$

where $E$, Young's modulus, is a measurement of the stiffness of an isotropic elastic material and the relationship between $E$, $\nu$, and $\mu$ is

$$
\nu=\frac{E}{2 \mu}-1 .
$$




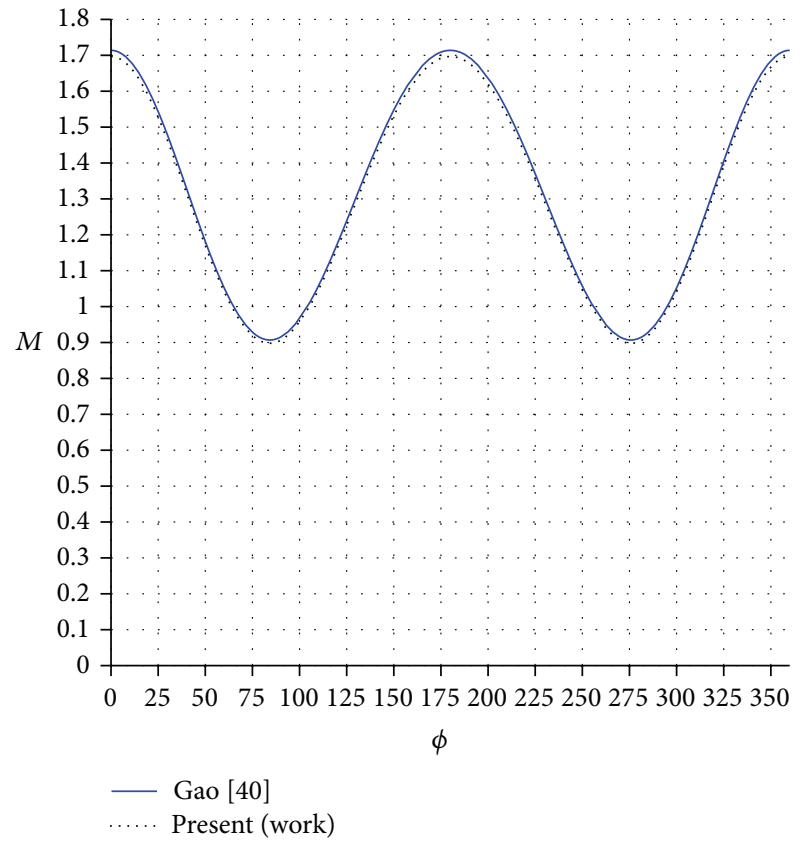

(a) $c=0.1$

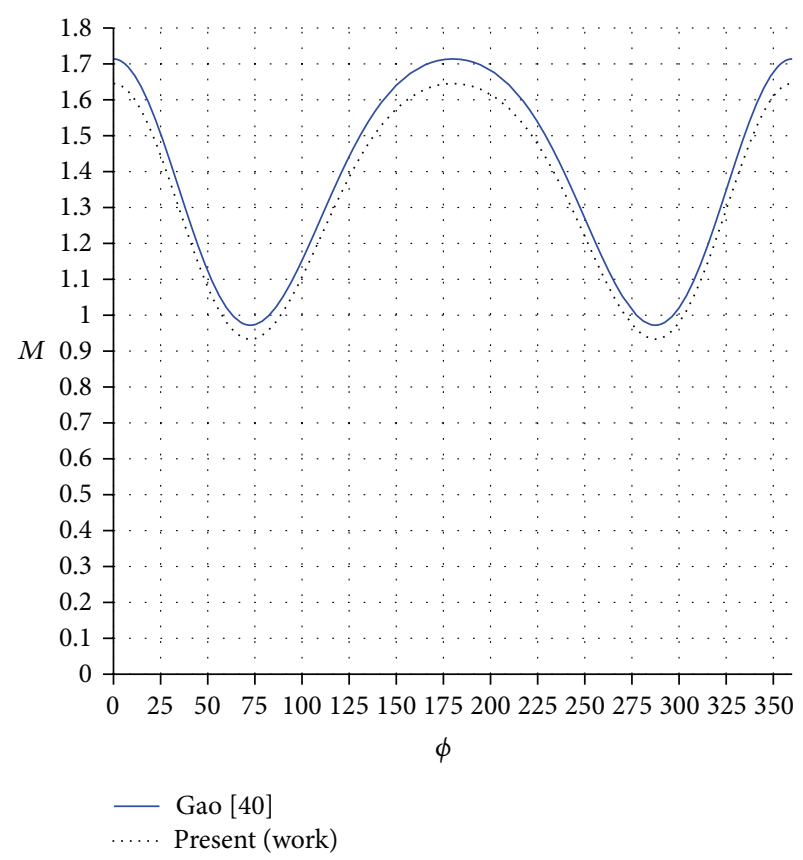

(b) $c=0.3$

Figure 5: The $M(\phi)$ for $f(\zeta)=\zeta+c \zeta^{2}$ at different $c$.

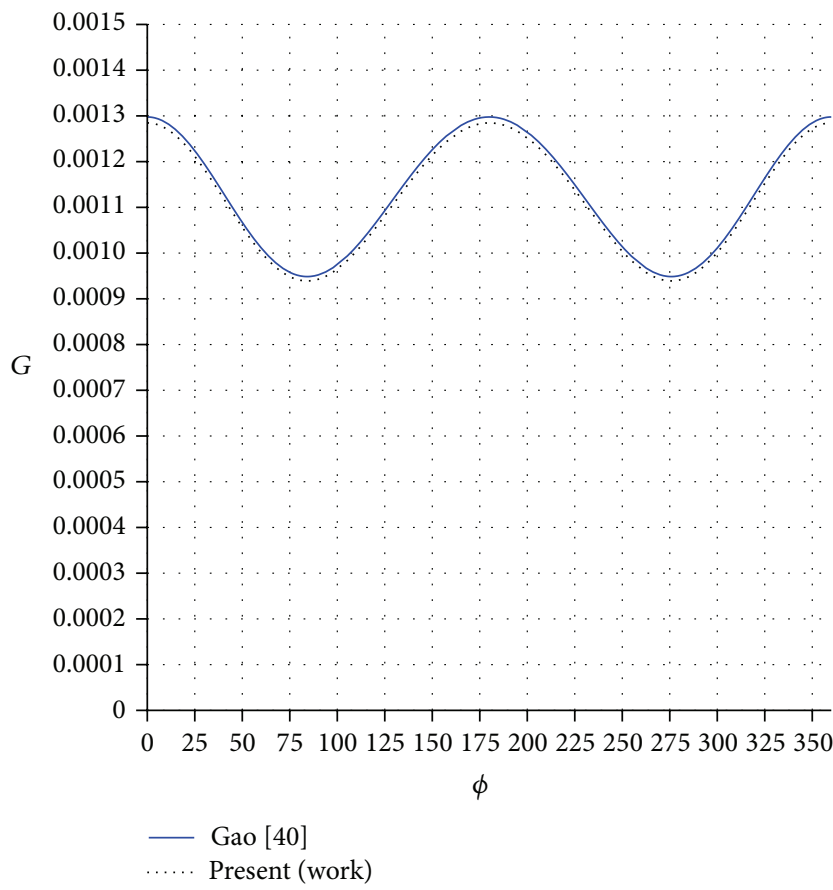

(a) $c=0.1$

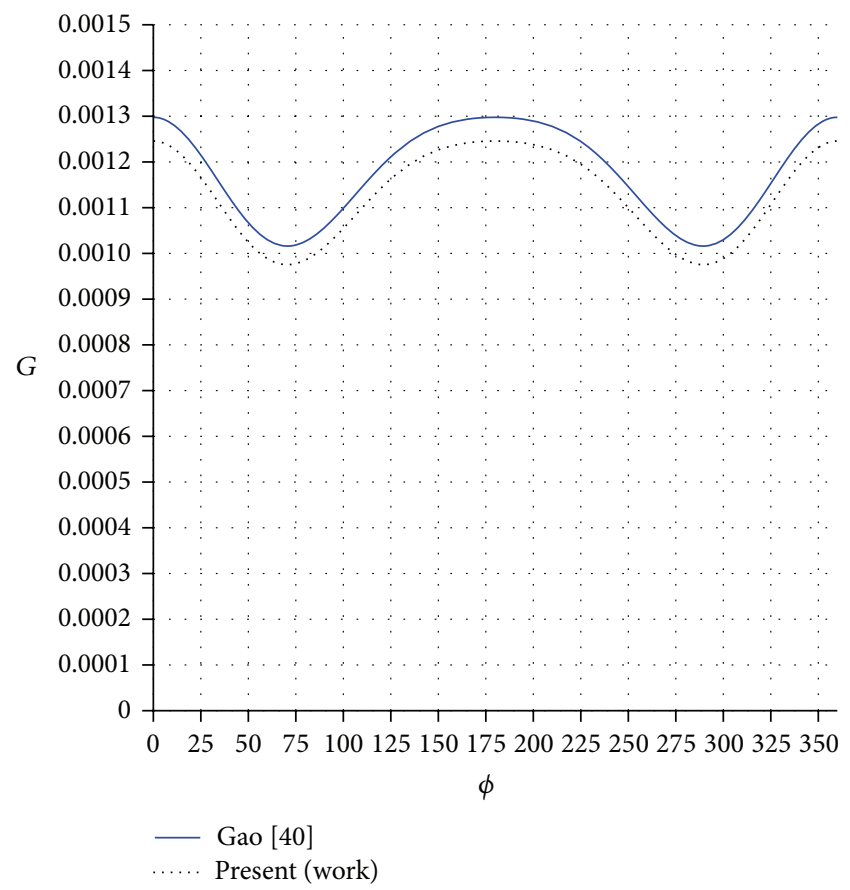

(b) $c=0.3$

Figure 6: The $G(\phi)$ for $f(\zeta)=\zeta+c \zeta^{2}$ at different $c$.

Let $a(\phi)=\left|f\left(e^{i \phi}\right)\right|$ and $r=\left|f\left(s e^{i \phi}\right)\right|$, followed by substituting $\quad$ where $\left|f\left(e^{i \phi}\right)-f\left(s e^{i \phi}\right)\right|=(1-s)\left|f^{\prime}\left(e^{i \phi}\right)\right|$ as $s \rightarrow 1$. Introduce (30) into (37), which leads to

$$
\begin{array}{rcc}
K_{j}(\phi)=V_{j} \lim _{s \rightarrow 1^{-}} \sqrt{\frac{2 \pi}{1-s}}\left|f^{\prime}(\zeta)\right|^{-1} \sum_{n, k} W_{k}^{n} A_{k}^{n}(s, \phi) ; & j=2,3, & Y_{k}^{n}(\phi)=D_{2 k+1}^{|n|+1 / 2}(0) \cos (n \phi), \\
(41) & C_{2 k+1}^{|n|+1 / 2}\left(\sqrt{1-s^{2}}\right)=\sqrt{1-s^{2}} D_{2 k+1}^{|n|+1 / 2}\left(\sqrt{1-s^{2}}\right),
\end{array}
$$




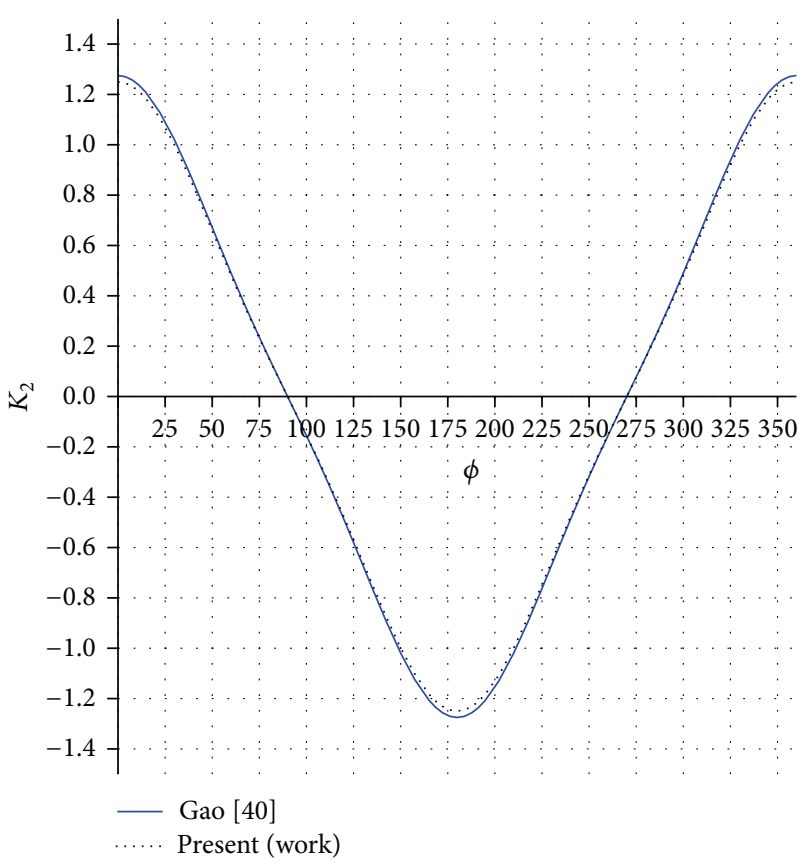

(a) $K_{2}(\phi)$

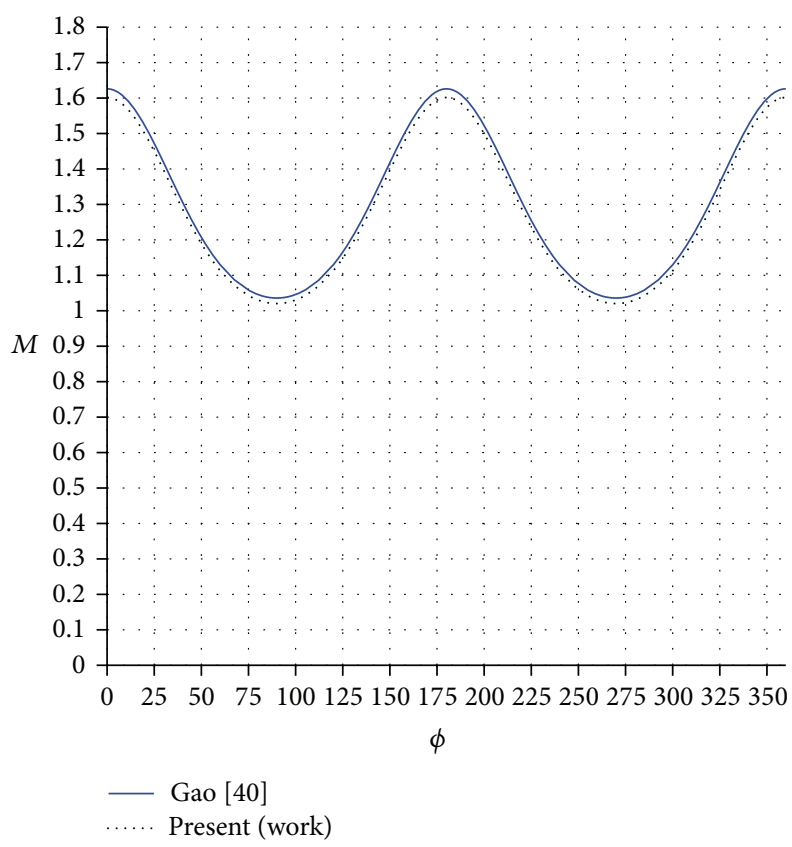

(c) $M(\phi)$

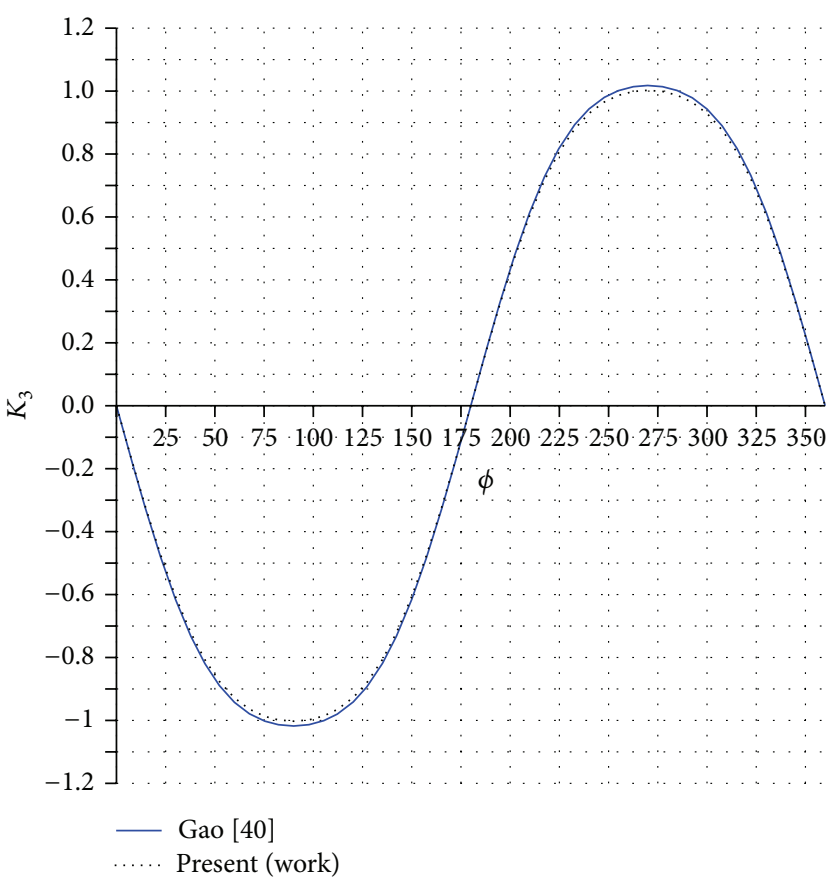

(b) $K_{3}(\phi)$

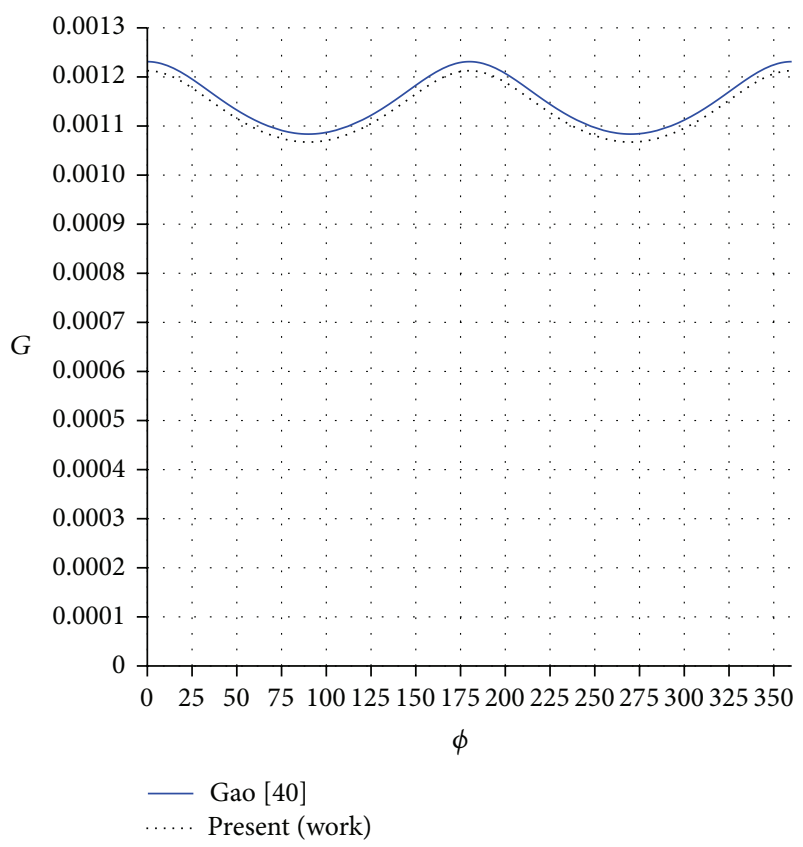

(d) $G(\phi)$

Figure 7: The $K_{2}(\phi), K_{3}(\phi), M(\phi)$, and $G(\phi)$ for $f(\zeta)=\zeta+c \zeta^{3}$ at $c=0.1$.

where $D_{m}^{\lambda}(x)$ is defined recursively by

$$
\begin{aligned}
m D_{m}^{\lambda}(x)= & 2(m+\lambda-1) x D_{m-1}^{\lambda}(x) \\
& -(m+2 \lambda-2) D_{m-2}^{\lambda}(x) ; \quad m=2,3,4, \ldots,
\end{aligned}
$$

with $D_{0}^{\lambda}(x)=2 \lambda$ and $D_{1}^{\lambda}(x)=2 \lambda x$. Substituting (42) into (41) yields

$$
K_{j}(\phi)=2 \sqrt{\pi} V_{j}\left|f^{\prime}\left(e^{i \phi}\right)\right|^{-1} \sum_{n, k} W_{k}^{n} Y_{k}^{n}(\phi),
$$

where the unknown coefficients, $W_{k}^{n}$, are obtained from (35). 


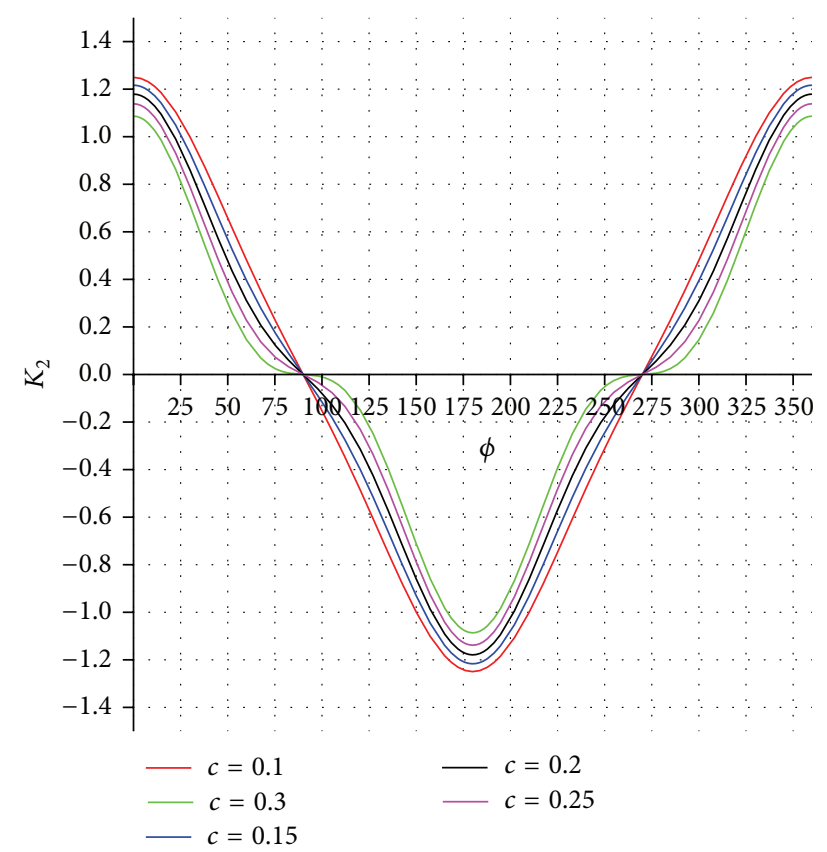

(a) $K_{2}(\phi)$

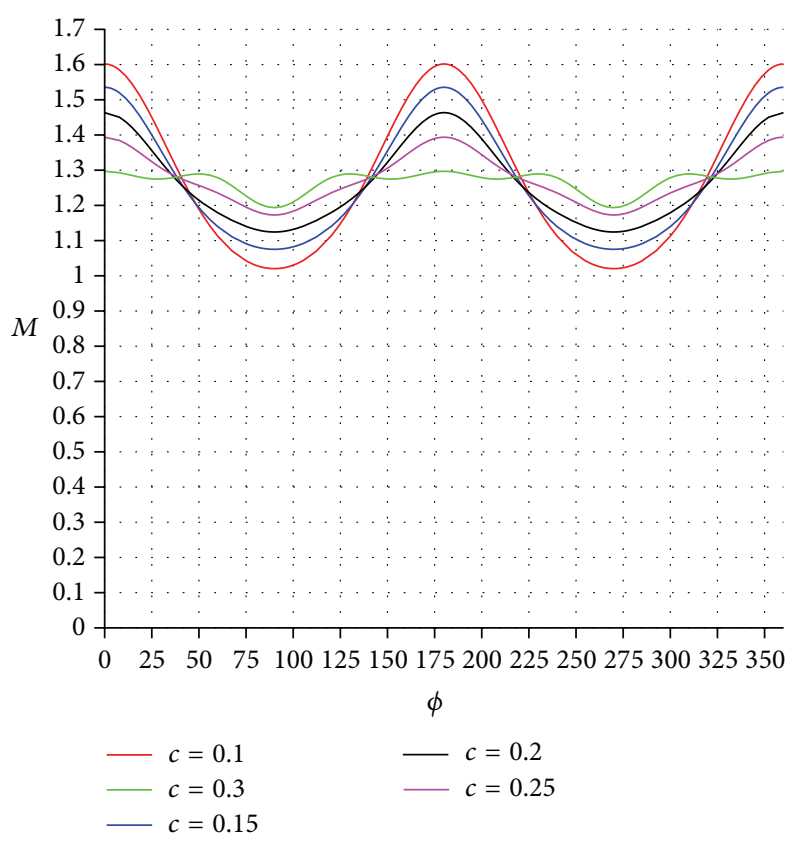

(c) $M(\phi)$

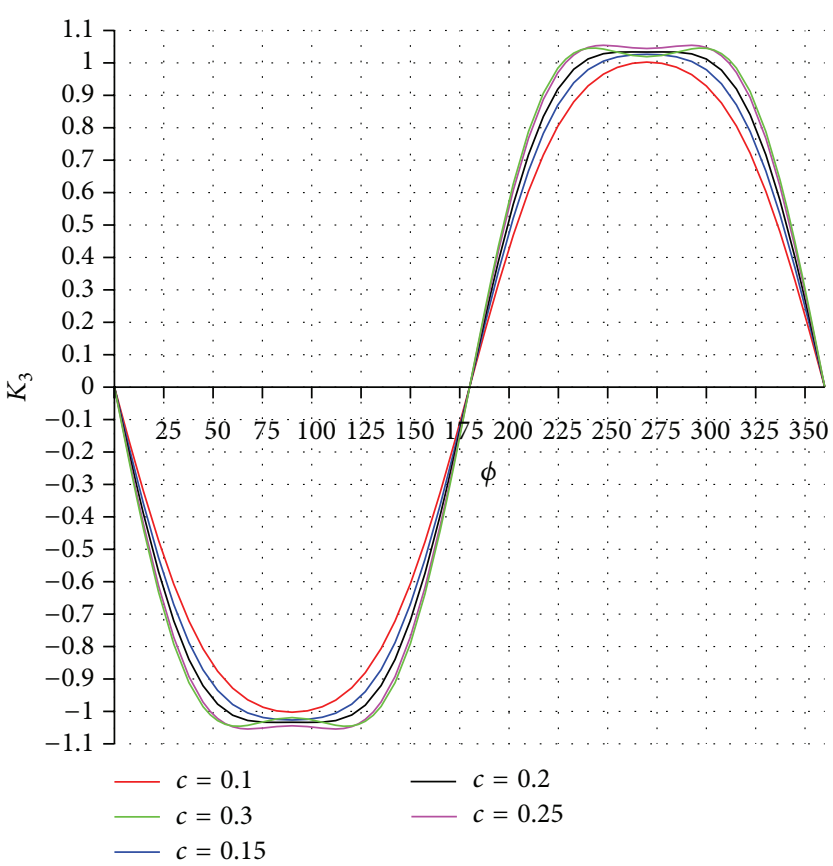

(b) $K_{3}(\phi)$

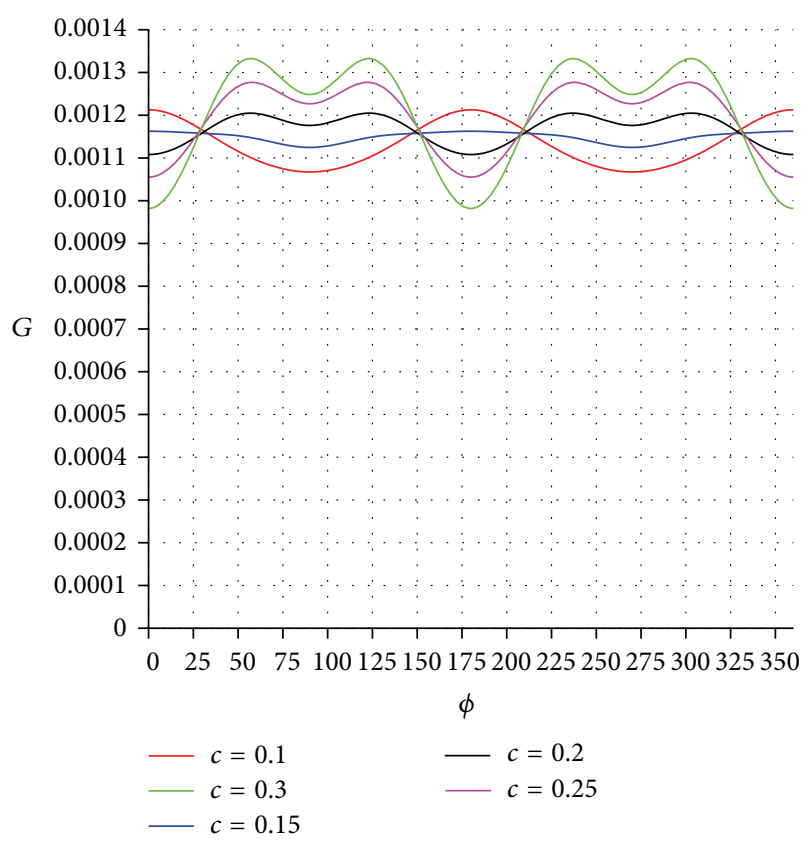

(d) $G(\phi)$

FIGURE 8: The $K_{2}(\phi), K_{3}(\phi), M(\phi)$, and $G(\phi)$ for $f(\zeta)=\zeta+c \zeta^{3}$ at various $c$.

\section{Results and Discussion}

Consider the conformal mapping [15]

$$
f(\zeta)=\zeta+c \zeta^{m+1} \text {, }
$$

where $m$ is an integer and $c$ must satisfy $-(1 / m) \leq c \leq(1 / m)$. The domain is circular if $c=0$ and has a smooth, regular boundary for $0 \leq(m+1)|c|<1$. As $(m+1)|c| \rightarrow 1$, one or more cusps develop; see Figure 2 for various $c$ and $m$, respectively.

Tables 1, 2, and 3 show that our numerical scheme converges rapidly at a different point of the crack with only a small value of $N=N_{1}=N_{2}$ used. Tables 1 and 2 show numerical scheme for $K_{2}(\phi)$ for $m=1$ with $c=0.1$ and $c=0.45$, respectively. Based on these two tables, it is evident that the convergence of stress intensity factors becomes slow 


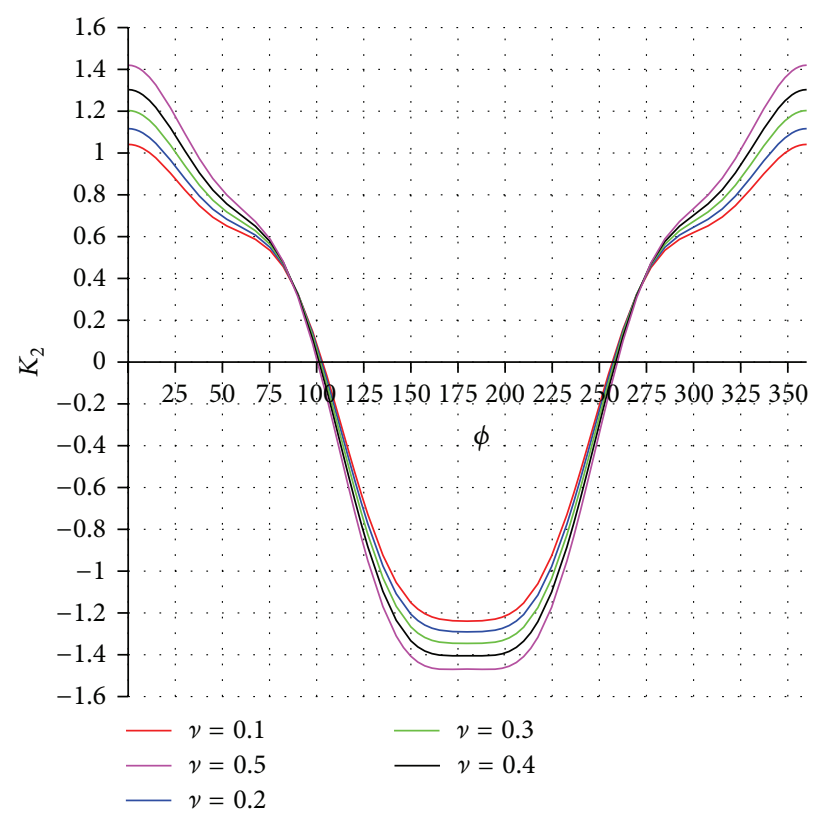

(a) $K_{2}(\phi)$

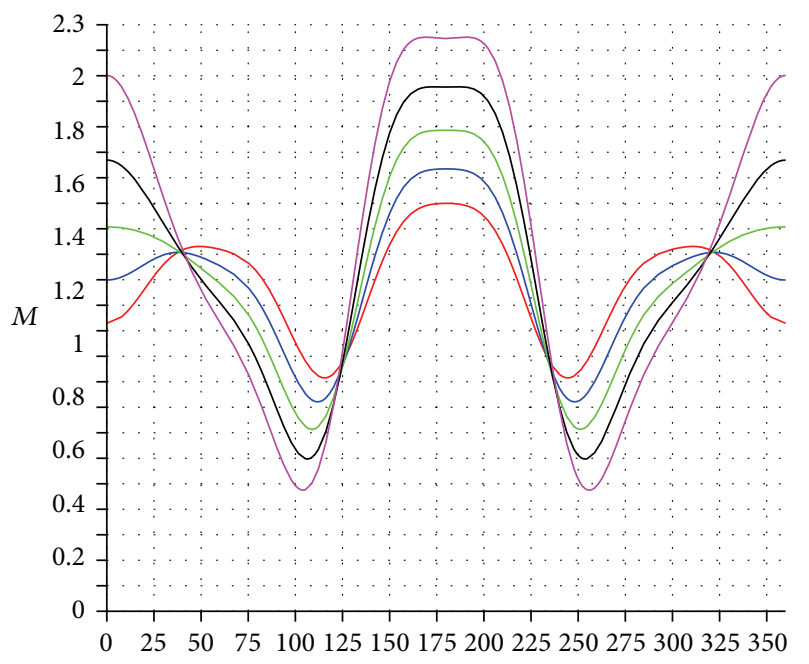

$\phi$

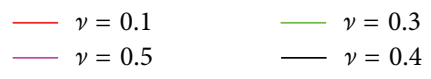

(c) $M(\phi)$

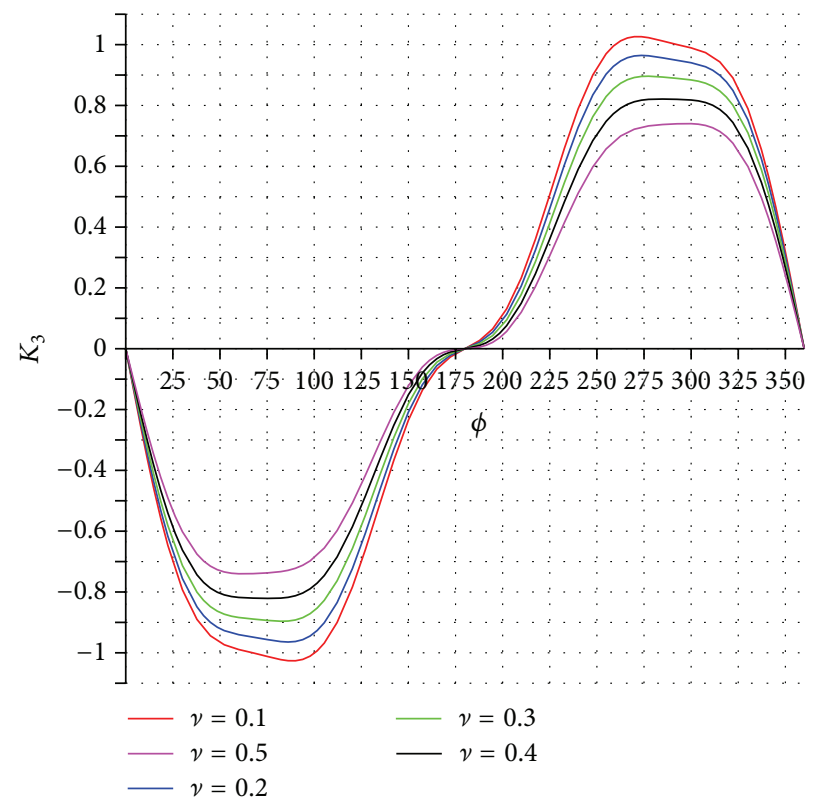

(b) $K_{3}(\phi)$

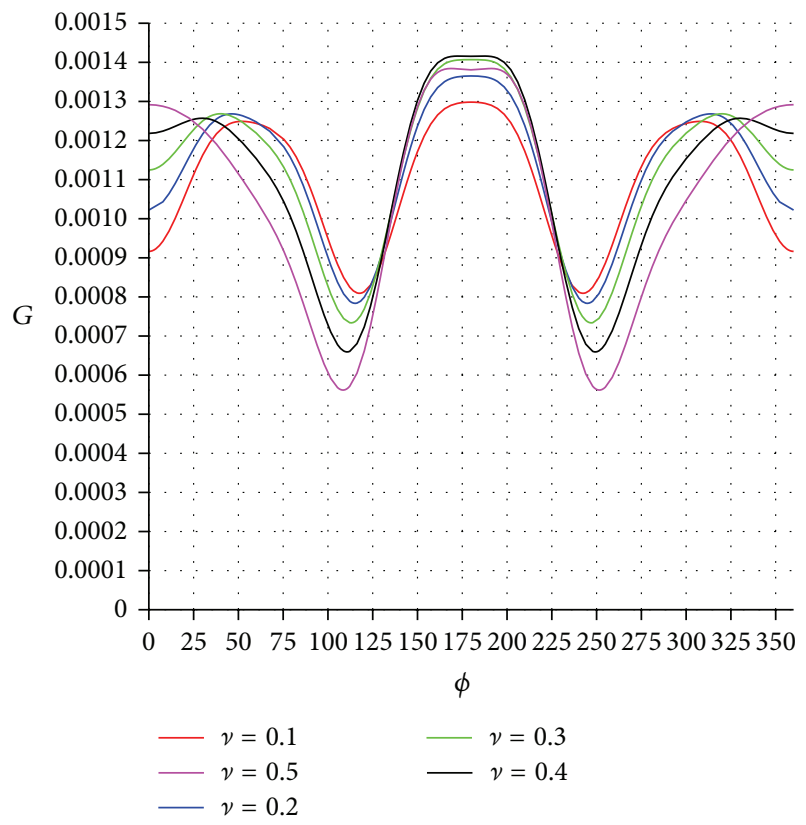

(d) $G(\phi)$

Figure 9: The $K_{2}(\phi), K_{3}(\phi), M(\phi)$, and $G(\phi)$ for $f(\zeta)=\zeta+0.1 \zeta^{4}$ at various $\nu$ with $\mu=1$.

as $c$ increases, whilst Table 3 presents the numerical scheme for $K_{3}(\phi)$, for $m=-2$ with $c=0.1$.

Figures 3, 4, 5, and 6 display the comparison of asymptotic and numerical solutions for $K_{2}(\phi)$ and $K_{3}(\phi)$ stress intensity factors, maximum stress intensity, $M(\phi)$, and strain energy release rate, $G(\phi)$, respectively, for $m=1$ at $c=0.1$ and $c=0.3$. As demonstrated in these figures, our results seem to agree with those obtained by Gao [40] except at the cusps. As the cusps become sharper, the analytical result by Gao
[40] does not work, and this gives rise to the difference between our and Gao's [40] result. It can be seen that the stress intensity factors have local extremal values when the crack front is at $\cos (\phi)= \pm 1$ or $\sin (\phi)= \pm 1$. Figure 7 presents the comparison of asymptotic and numerical solutions for $K_{2}(\phi)$ and $K_{3}(\phi)$ stress intensity factors, maximum stress intensity, $M(\phi)$, and strain energy release rate, $G(\phi)$, respectively, for $m=2$ at $c=0.1$. Figure 8 shows the variations of $K_{2}, K_{3}$, $M$, and $G$ against $\phi$ for various of $c \leq 0.3$. Similar behavior 
can be observed for the solution of $K_{2}(\phi), K_{3}(\phi), M(\phi)$, and $G(\phi)$, for a different parameter of $\nu$ for $f(\zeta)+0.1 \zeta^{4}$, displayed in Figure 9.

\section{Conclusion}

The present work dealt with the epicycloid crack with the application of shear loading in fracture mechanics. To this end, the numerical solution for the stresses in such specimens is derived, based on the solution of the hypersingular integral equation, and the conformal mapping technique is adopted to transform the hypersingular integral equation over a circular region such that the equation is reduced into a system of linear equations and solved for the unknown coefficients. The stress intensity factors, maximum stress intensity, and strain energy release rate for the epicycloid crack subject to shear load are presented graphically. The proposed model and the obtained numerical results are in good agreement when compared to Gao [40].

\section{Conflict of Interests}

The authors declare that there is no conflict of interests regarding the publication of this paper.

\section{Acknowledgment}

This project is supported by the Universiti Putra Malaysia for the Research University Grant scheme Project no. 05-02-121834RU.

\section{References}

[1] C. M. Segedin, "Note on a penny-shaped crack under shear," Mathematical Proceedings of the Cambridge Philosophical Society, vol. 47, no. 2, pp. 396-400, 1951.

[2] H. D. Bui, "An integral equations method for solving the problem of a plane crack of arbitrary shape," Journal of the Mechanics and Physics of Solids, vol. 25, no. 1, pp. 29-39, 1977.

[3] H. Ishikawa, "A finite element analysis of stress intensity factors for combined tensile and shear loading by only a virtual crack extension," International Journal of Fracture, vol. 16, no. 5, pp. R243-R246, 1980.

[4] M. A. Astiz, "An incompatible singular elastic element for twoand three-dimensional crack problems," International Journal of Fracture, vol. 31, no. 2, pp. 105-124, 1986.

[5] H. G. Boem and Y. Y. Earmme, "Analysis of a at annular crack under shear loading," KSME Iinternational Journal of Mechanical Science and Technology, vol. 7, no. 1, pp. 35-47, 1993.

[6] Y.Z. Chen, "Stress intensity factors for curved and kinked cracks in plane extension," Theoretical and Applied Fracture Mechanics, vol. 31, no. 3, pp. 223-232, 1999.

[7] C. W. Kuang, "A new boundary integral equation method for analysis of cracked linear elastic bodies," Journal of the Chinese Institute of Engineers, vol. 27, no. 6, pp. 937-941, 2004.

[8] E. Atroshchenko, S. Potapenko, and G. Glinka, "Stress intensity factor for an embedded elliptical crack under arbitrary normal loading," International Journal of Fatigue, vol. 31, no. 11-12, pp. 1907-1910, 2009.
[9] L. Banks-Sills, "Update: application of the finite element method to linear elastic fracture mechanics," Applied Mechanics Reviews, vol. 63, no. 2, Article ID 020803, pp. 1-17, 2010.

[10] N. M. A. N. Long and M. Yaghobifar, "General analytical solution for stress intensity factor of a hypocycloid hole with many cusps in an infinite plate," Philosophical Magazine Letters, vol. 91, no. 4, pp. 256-263, 2011.

[11] I. N. Sneddon, "The distribution of stress in the neighbourhood of a crack in an elastic solid," Proceedings of the Royal Society A: Mathematical, Physical and Engineering Sciences, vol. 187, no. 1009, pp. 229-260, 1946.

[12] M. K. Kassir, "Stress-intensity factor for a three dimensional rectangular crack," Journal of Applied Mechanics, vol. 48, no. 2, pp. 309-312, 1981.

[13] M. K. Kassir, "A three-dimensional rectangular crack subjected to shear loading," International Journal of Solids and Structures, vol. 18, no. 12, pp. 1075-1082, 1982.

[14] N. I. Ioakimidis, "Two methods for the numerical solution of Bueckner's singular integral equation for plane elasticity crack problems," Computer Methods in Applied Mechanics and Engineering, vol. 31, no. 2, pp. 169-177, 1982.

[15] N. I. Muskhelishvili, Some Basic Problems of the Mathematical Theory of Elasticity, Noordhoff International Publishing, Leyden, The Netherlands, 1975.

[16] B. Cotterell and J. R. Rice, "Slightly curved or kinked cracks," International Journal of Fracture, vol. 16, no. 2, pp. 155-169, 1980.

[17] W.-L. Wu, "Dual boundary element method applied to antiplane crack problems," Mathematical Problems in Engineering, vol. 2009, Article ID 132980, 10 pages, 2009.

[18] H. Georgiadis and P. Gourgiotis, "An approach based on integral equations for crack problems in standard couple-stress elasticity," in Mechanics of Generalized Continua, vol. 21 of Advances in Mechanics and Mathematics, chapter 26, pp. 253262, Springer Science Business Media, 2010.

[19] P. Lazzarin and M. Zappalorto, "A three-dimensional stress field solution for pointed and sharply radiused $\mathrm{V}$-notches in plates of finite thickness," Fatigue \& Fracture of Engineering Materials \& Structures, vol. 35, no. 12, pp. 1105-1119, 2012.

[20] P. Lazzarin, M. Zappalorto, and F. Berto, "Recent developments in multi-parametric three-dimensional stress field representation in plates weakened by cracks and notches," Frattura ed Integrità Strutturale, vol. 7, no. 25, pp. 61-68, 2013.

[21] N. I. Ioakimidis, "Application of finite-part integrals to the singular integral equations of crack problems in plane and three-dimensional elasticity," Acta Mechanica, vol. 45, no. 1-2, pp. 31-47, 1982.

[22] T.-Y. Qin and R.-J. Tang, "Finite-part integral and boundary element method to solve flat crack problems," Applied Mathematics and Mechanics, vol. 13, no. 12, pp. 1089-1095, 1992.

[23] A. M. Linkov and S. G. Mogilevskaya, "Complex hypersingular integrals and integral equations in plane elasticity," Acta Mechanica, vol. 105, no. 1-4, pp. 189-205, 1994.

[24] P. A. Martin, "Mapping flat cracks onto penny-shaped cracks, with application to somewhat circular tensile cracks," Quarterly of Applied Mathematics, vol. 54, no. 4, pp. 663-675, 1996.

[25] Y. Z. Chen and K. Y. Lee, "Numerical solution of threedimensional crack problem by using hypersingular integral equation," Computer Methods in Applied Mechanics and Engineering, vol. 190, no. 31, pp. 4019-4026, 2001.

[26] E. E. Theotokoglou, "Boundary integral equation method to solve embedded planar crack problems under shear loading," Computational Mechanics, vol. 33, no. 5, pp. 327-333, 2004. 
[27] J. T. Guidera and R. W. Lardner, "Penny-shaped cracks," Journal of Elasticity, vol. 5, no. 1, pp. 59-73, 1975.

[28] P. A. Martin, "The discontinuity in the elastostatic displacement vector across a penny-shaped crack under arbitrary loads," Journal of Elasticity, vol. 12, no. 2, pp. 201-218, 1982.

[29] P. A. Martin, "Mapping flat cracks onto penny-shaped cracks: shear loadings," Journal of the Mechanics and Physics of Solids, vol. 43, no. 2, pp. 275-294, 1995.

[30] N. I. Ioakimidis, "Upper bounds for the stress intensity factors along the boundaries of interacting coplanar cracks in threedimensional elasticity," Engineering Fracture Mechanics, vol. 16, no. 6, pp. 821-826, 1982.

[31] P. A. Martin, "Exact solution of a simple hypersingular integral equation," Journal of Integral Equations and Applications, vol. 4, no. 2, pp. 197-204, 1992.

[32] P. A. Martin, "On wrinkled penny-shaped cracks," Journal of the Mechanics and Physics of Solids, vol. 49, no. 7, pp. 1481-1495, 2001.

[33] N. I. Ioakimidis, “Two-dimensional principal value hypersingular integrals for crack problems in three-dimensional elasticity," Acta Mechanica, vol. 82, no. 1-2, pp. 129-134, 1990.

[34] J. Hadamard, Lectures on Cauchy's Problem in Linear Partial Differential Equations, Dover Publications, New York, NY, USA, 2003.

[35] H. D. Bui, "Application des potentiels ealastiques aletude des fissures planes de forme arbitraire en milieu tridimensionnel," Comptes Rendus Mathematique: Académie des Sciences A, vol. 280, pp. 1157-1160, 1975.

[36] Z. Nehari, Conformal Mapping, McGraw-Hill, New York, NY, USA, 1st edition, 1952.

[37] I. S. Gradshteyn and I. M. Ryzhik, Table of Integrals, Series, and Products, Academic Press, Boston, Mass, USA, 5th edition, 1994.

[38] S. Krenk, "A circular crack under asymmetric loads and some related integral equations," Journal of Applied Mechanics, Transactions ASME, vol. 46, no. 4, pp. 821-826, 1979.

[39] H. Gao and J. R. Rice, "Shear stress intensity factors for a planar crack with slightly curved front," Journal of Applied Mechanics, Transactions ASME, vol. 53, no. 4, pp. 774-778, 1986.

[40] H. Gao, "Nearly circular shear mode cracks," International Journal of Solids and Structures, vol. 24, no. 2, pp. 177-193, 1988. 


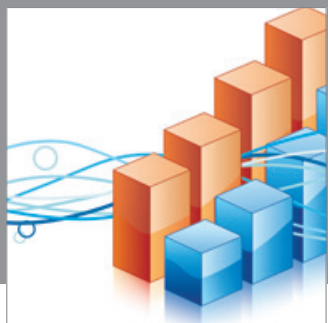

Advances in

Operations Research

mansans

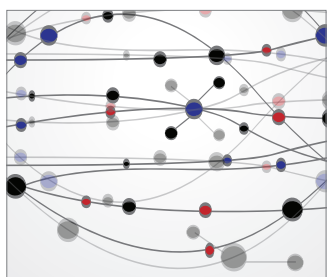

The Scientific World Journal
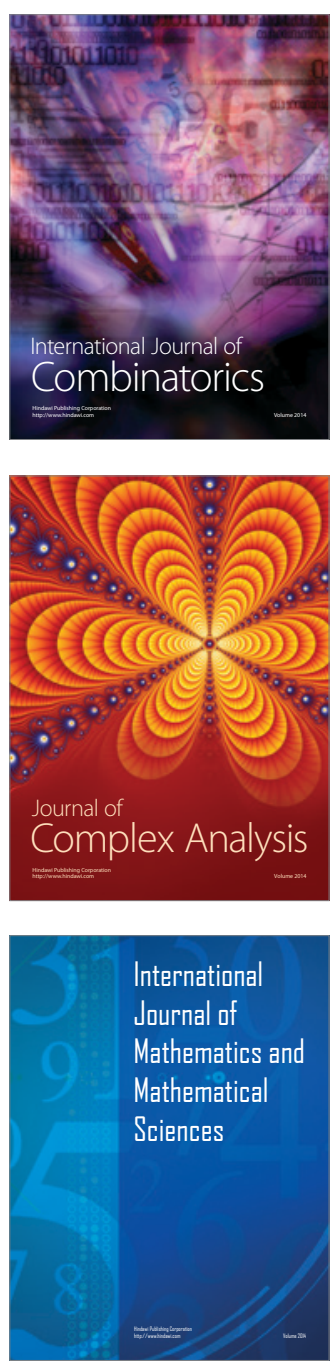
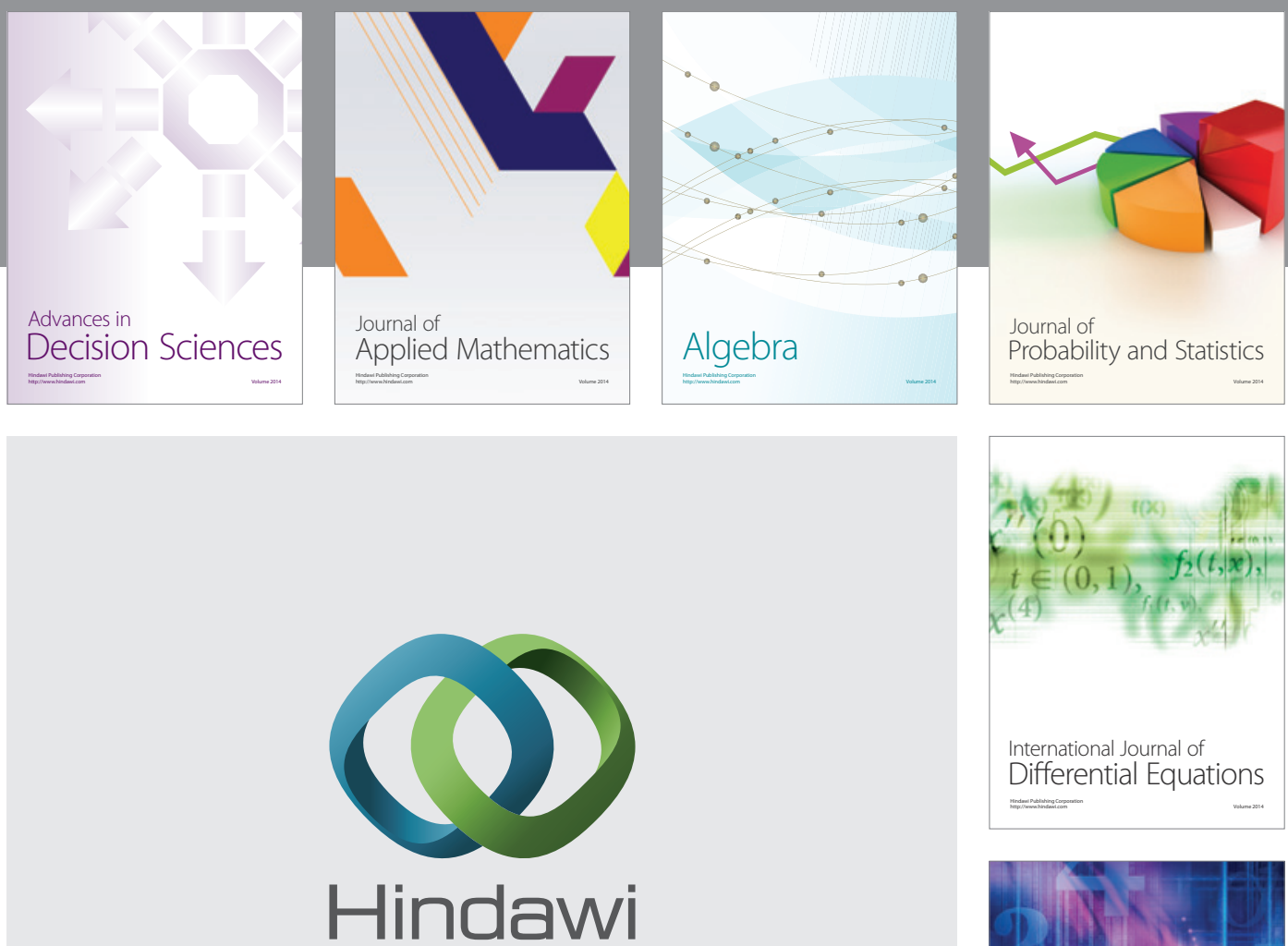

Submit your manuscripts at http://www.hindawi.com
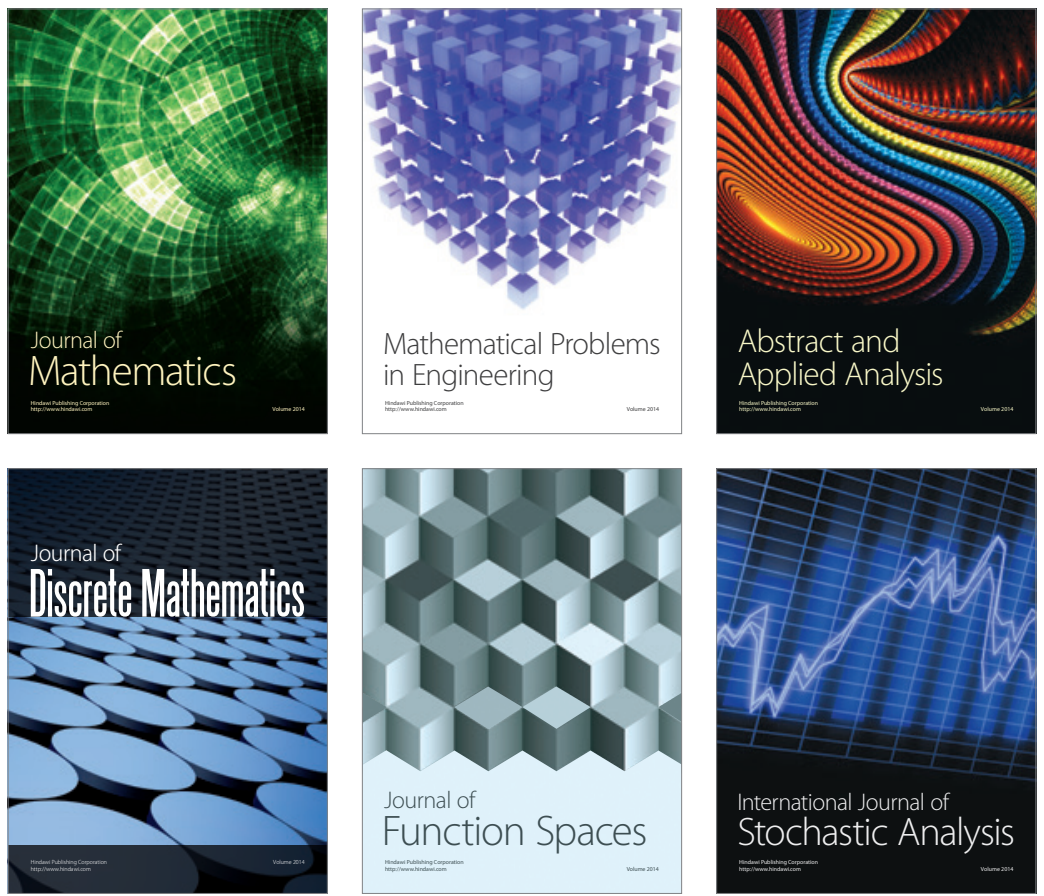

Journal of

Function Spaces

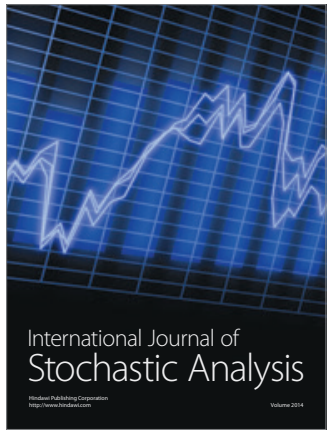

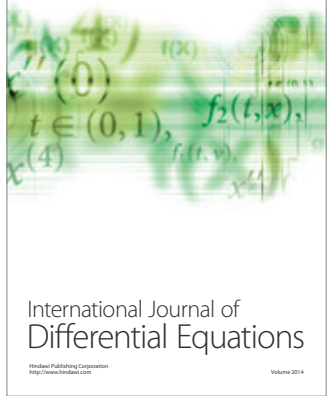
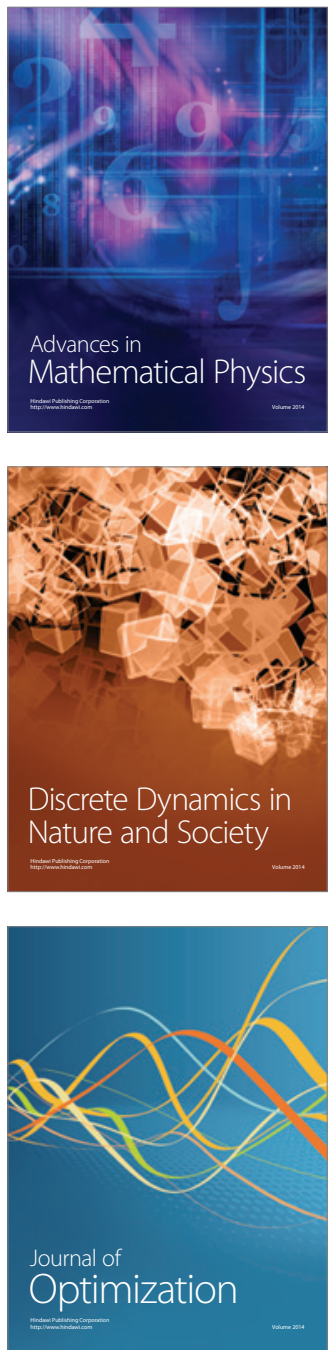